\title{
MODELAGEM DO PROCESSO DE GRANULAÇÃO DE POLVILHO DOCE EM LEITO FLUIDIZADO PULSADO POR BALANÇO POPULACIONAL
}

\author{
Feltre, G. ${ }^{1}$, Hirata, T. A. M. ${ }^{2}$, Menegalli, F.C. ${ }^{2}$, Dacanal, G. C. ${ }^{1}$ \\ 1 Dep. de Eng. de Alimentos, Faculdade de Zootecnia e Engenharia de Alimentos, USP \\ 2 Dep. de Eng. de Alimentos, Faculdade de Engenharia de Alimentos, UNICAMP \\ *e-mail: gdacanal@usp.br
}

\begin{abstract}
RESUMO - O processo de aglomeração em leito fluidizado pulsado pode ser utilizado para aumentar o tamanho de partículas de pós finos e coesivos. Os grânulos produzidos normalmente possuem maior fluidez e dispersão em líquidos. Neste trabalho foi realizada a modelagem matemática deste sistema por equações de balanço populacional. A partir das distribuiçõos de tamanho das partículas, realizou-se a o ajuste do modelo por meio de equações de balanço populacional, a fim de se obter a constante de coalescência $(\beta 0)$. Os processos foram realizados por bateladas com $0,40 \mathrm{~kg}$ de pó, em $50 \mathrm{~min}$. A pulsação do ar foi obtida pela rotação de uma válvula esfera nas frequências de $(300,600$ e 900) rpm. O tamanho dos grânulos aumentou de $57 \mu \mathrm{m}$ para $(534,505$ e 438) $\mu \mathrm{m}$, respectivamente. A qualidade da fluidização foi melhorada com a pulsação do ar e resultou em maior homogeneidade do leito. O maior valor da constante $(\beta 0)$ foi obtida a $300 \mathrm{rpm}$, que foi a condição operacional que produziu grânulos com maior tamanho.
\end{abstract}

\section{INTRODUÇÃO}

O crescimento das partículas durante processos de aglomeração pode ser descrito pelo modelo de balanço populacional (MBP), que se fundamenta nos mecanismos de colisões entre partículas. Diversos autores trabalharam com modelos baseados em balanços populacionais para simulação de processos de granulação, por esses modelos apresentarem bons resultados e apresentarem alta eficiência computacional (PEGLOW et al., 2007; TAN et al., 2004; TAN et al., 2006; LIU e LITSTER, 2002). Mais especificamente, os modelos de balanço populacional são balanços numéricos em torno de cada intervalo da distribuição de tamanho de partículas, baseado na conservação do número de partículas. Deste modo, é possível descrever como a taxa de variação no número de partículas pode ser relacionada com a taxa na qual as partículas entram ou saem daquele intervalo de tamanho, por diferentes fenômenos que ocorrem no sistema (nucleação, agregação, quebra, etc.). $\mathrm{O}$ MBP, discretizado, é representado pela Equação 1 (RAMKRISHNA, 2000; HOUNSLOW et al., 2001; CAMERON et al., 2005). A maneira mais usual de resolução por discretização foi a proposta por HOUNSLOW et al (1988), por sua simplicidade e eficiência. 
$\frac{d N_{i}}{d t}=N_{(i-1)} \sum_{j=1}^{\mathrm{i}-2} 2^{(i-j+1)} \beta_{(i-1, j)} N_{j}+\frac{1}{2} \beta_{(i-1, i-1)} N_{(i-1)}^{2}$

$-\mathrm{N}_{\mathrm{i}} \sum_{\mathrm{j}=1}^{\mathrm{i}-1} 2^{\mathrm{i}-\mathrm{j}} \beta_{(\mathrm{i}, \mathrm{j})} \mathrm{N}_{\mathrm{j}}-\mathrm{N}_{\mathrm{i}} \sum_{\mathrm{j}=1}^{\mathrm{i} \max } \beta_{(\mathrm{i}, \mathrm{j})} \mathrm{N}_{\mathrm{j}}$

$\mathrm{i}=1,2, \ldots, \mathrm{i}_{\max }$

em que,

$\beta_{(\mathrm{i}, \mathrm{j})}=\beta 0\left|V_{i}-V_{j}\right|$

O "kernel" de coalescência $\beta$ representa a frequiência de colisões entre as partículas de tamanho i e j, bem-sucedidas em formar uma partícula de tamanho " $\mathrm{i}+\mathrm{j}$ ".

\section{MATERIAIS E MÉTODOS}

\subsection{Amido de mandioca (polvilho)}

Os ensaios em batelada utilizaram como matéria-prima amostras com 0,40 $\mathrm{kg}$ de amido de mandioca, ou polvilho doce (YOKI Alimentos S.A., Brasil), obtidas comercialmente. A composição química do polvilho doce consiste de $0,4 \%$ de proteínas, $0,2 \%$ de fibras, $13,1 \%$ de umidade, $86,1 \%$ de carboidratos e $0,2 \%$ de cinzas.

O agente ligante usado no processo de aglomeração foi solução aquosa de maltodextrina (20\%DE, MOR-REX® ${ }^{\circledR} 1920$, Corn Products, Brazil), a $27^{\circ} \mathrm{C}$ e concentração de $50 \%$ w./w.

\subsection{Equipamento}

O equipamento usado foi um leito fluidizado cônico pulsado operado em batelada, localizado no Laboratório de Operações Unitárias do Departamento de Engenharia de Alimentos da FZEA/USP. O leito é constituído por uma base cônica de 4" de diâmetro por $150 \mathrm{~mm}$ de altura, e um corpo cilíndrico de 10" de diâmetro por $400 \mathrm{~mm}$ de altura (Figura 1).

O aquecimento do ar fluidizante foi realizado por resistências elétricas. A vazão do ar fluidizante foi monitorada por uma placa orifício previamente calibrada por um anemômetro de fio quente. As partículas elutriadas foram coletadas por um ciclone instalado na saída de ar do leito fluidizado. O sistema de pulsação do ar foi realizado por meio da rotação de uma válvula esfera, instalada abaixo da placa distribuidora de ar. A frequência de rotação da válvula, em rotações por minuto, é equivalente ao número de ciclos por minuto de abertura/fechamento da corrente de ar fluidizante. 


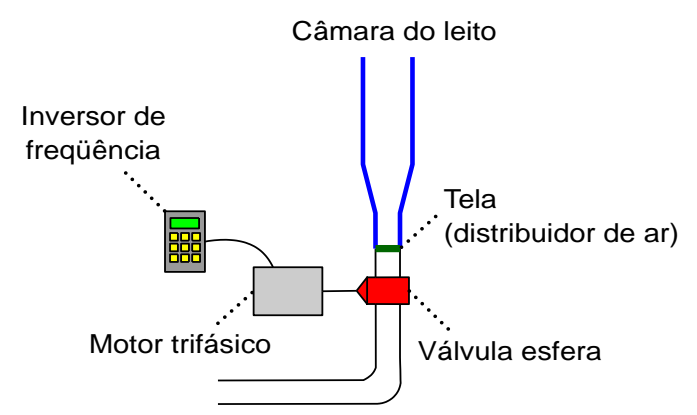

Figura 1 - Esquema do sistema de pulsação com válvula esfera (Dacanal et. al., 2010).

\subsection{Fluidodinâmica em leito pulsado}

A primeira etapa deste trabalho consiste no estudo da fluidodinâmica de amido de mandioca em leito fluidizado cônico pulsado, a fim de verificar os efeitos do uso de diferentes frequências de pulsação durante a fluidização destes particulados. $\mathrm{O}$ volume de amostra utilizado em cada batelada foi igual a $0,40 \mathrm{~kg}$. Por fim, as curvas de fluidização foram obtidas variando-se a frequência de pulsação em 0 (sem pulsação), 300, 600 e 900 rpm

\subsection{Aglomeração de polvilho doce em leito pulsado}

Para a aglomeração do polvilho doce em diferentes frequências de pulsação, o tempo de aglomeração foi limitado em até 50 minutos de processo, mantendo-se a temperatura e velocidade de fluidização em $95{ }^{\circ} \mathrm{C}$ e $0.30 \mathrm{~m} / \mathrm{s}$. A pressão de atomização, altura do bico aspersor (modelo $1 / 8 \mathrm{JN}$ SS+SU11-SS, Spraying Systems, Brazil) e massa de amostra foram fixados em 0,5 bar, $300 \mathrm{~mm}$ e $0,40 \mathrm{~kg}$, respectivamente. A vazão de ligante atomizado foi mantida em 3,0 g/min.

\subsection{Diâmetro médio de partículas e distribuição de tamanho}

O tamanho das partículas foi obtido por meio de análises de imagem por microscopia. As partículas das amostras foram distribuídas sobre uma lâmina de vidro e observadas por um estereomicroscópio (Stereo Discovery V8, Zeiss, Germany), equipado com uma câmera digital (Axiocam ICc 3, Zeiss). O diâmetro médio e distribuição de tamanho das partículas foram determinados por meio do diâmetro equivalente (Equação 3), que é referente à área de projeção 2D das partículas. Os diâmetros equivalentes foram classificados em diferentes intervalos de tamanho, em intervalos proporcionais a $\sqrt[3]{2}$. Posteriormente, foi obtida a distribuição de tamanho utilizando-se os intervalos de tamanho entre (1 a 3500) $\mu \mathrm{m}$. As partículas com tamanho menor a $1 \mu \mathrm{m}$ foram desconsideradas, a fim de eliminar possíveis erros gerados pelos filtros de imagem. O diâmetro médio de partículas ( $D 4.3$ ) foi calculado baseando-se na fração numérica $\left(f_{i}\right)$ contida em cada diâmetro equivalente ( $d p_{e q i}$ ) classificado no intervalo “ $i$ ”, como descrito pela Equação 2. 


$$
\begin{aligned}
d p_{e q} & =\sqrt{\frac{4 S}{\pi}} \\
D 4.3 & =\frac{\sum f_{i} d p_{e q i}^{4}}{\sum f_{i} d p_{e q i}^{3}}
\end{aligned}
$$

\subsection{Modelagem matemática}

A modelagem matemática foi realizada pelo ajuste e obtenção dos valores do kernel, considerado constante, ou seja, $\beta_{(\mathrm{i}, \mathrm{j})}=\beta 0\left|\mathrm{~V}_{\mathrm{i}}-\mathrm{V}_{\mathrm{j}}\right|$. Para a resolução das equações de balanço populacional, foi utilizado o software MATLAB v.R2009a. A resolução numérica do sistema de equações diferenciais ordinárias (EDO) foi feita pelo pacote ODE45 (método de Runge-Kutta de $4 \mathrm{e}$ $5^{\mathrm{a}}$ ordem). $\mathrm{O}$ número de intervalos de tamanho de partículas foi $\mathrm{i}_{\max }=16$. Para o ajuste estatístico das constantes $\beta_{0}$, foi utilizado o pacote de otimização LSQNONLIN do MATLAB.

\section{RESULTADOS E DISCUSSÃO}

A Figura 2 mostra o diâmetro médio de partícula. Pode-se observar que o diâmetro médio de partículas (D4.3) da matéria-prima e do produto aglomerado aumentou de $57,5 \mu \mathrm{m}$ a $(533,9,504,7$ e $438,0) \mu \mathrm{m}$, de acordo com a frequência de pulsação de 300, 600 e $900 \mathrm{rpm}$, respectivamente.

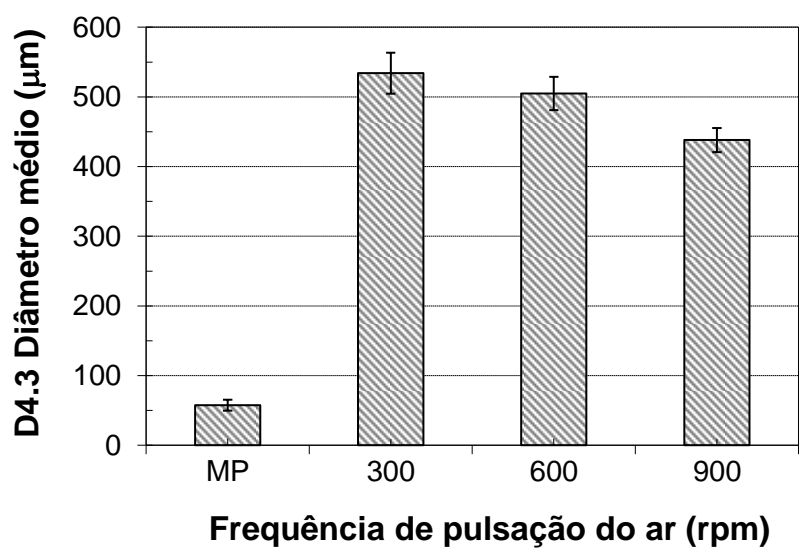

Figura 2 - Diâmetro médio de particulas (de Brouckere).

A Figura 3 apresenta os resultados obtidos para a análise de distribuição de tamanho de partículas para o amido de mandioca "in natura" e grânulos produzidos a (300, 600 e 900) rpm. Podese visualizar que a distribuição de tamanho de partículas para os produtos aglomerados se diferenciam, devido à redução da fração de partículas finas e formação de grânulos com tamanho superior a $50 \mu \mathrm{m}$. 


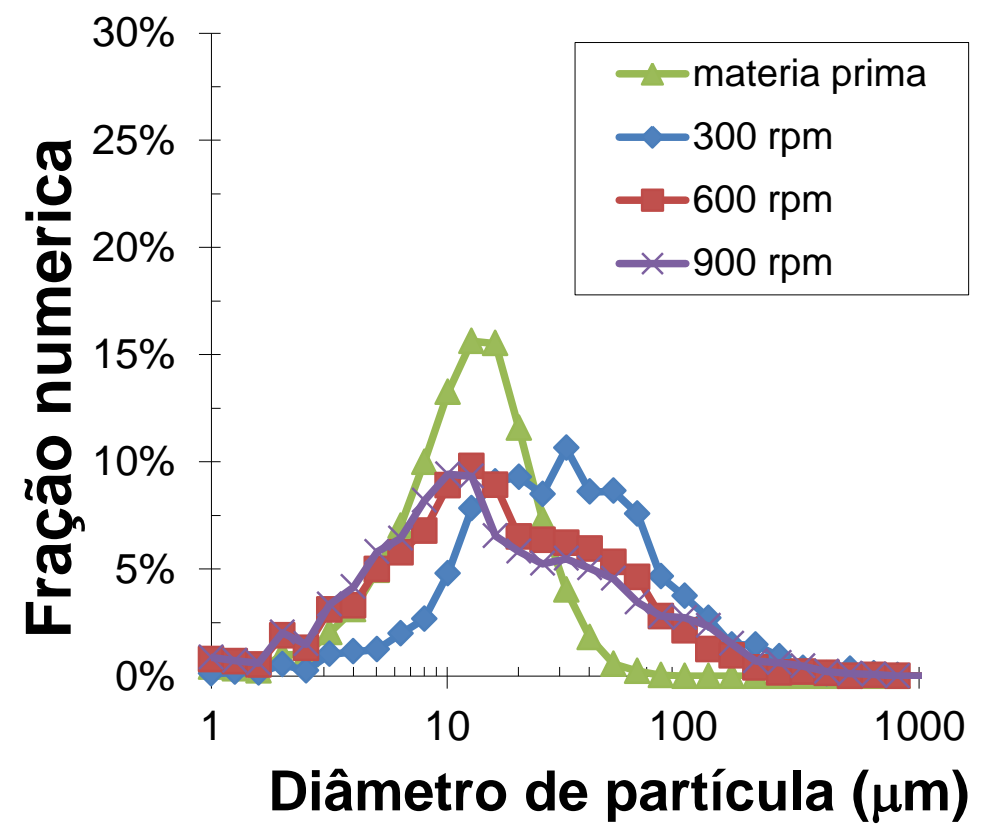

Figura 3 - Distribuição de tamanho de partículas para o polvilho doce "in natura" e aglomerado nas frequências de pulsação de (300, 600 e 900) rpm.

Os dados de distribuição de tamanho foram ajustados utilizando-se o modelo de balanço populacional, em que se extraiu os valores de $\beta 0$, para o kernel de coalescência $\beta 0(\mathrm{Vi}-\mathrm{Vj})$. Os valores de $\beta 0$ representam a taxa de aglomeração (Tabela 1 ), ou seja, maiores valores de $\beta 0$ são resultantes de processos de mais eficazes e sem quebra.

Tabela 1: Valores de $\beta 0$ obtidos a partir dos processos de aglomeração em leito fluidizado pulsado.

\begin{tabular}{|c|c|}
\hline Frequência de pulsação & Valor de $\boldsymbol{\beta 0}\left(\mathbf{m i n}^{-1}\right)$ \\
\hline $300 \mathrm{rpm}$ & 0,1958 \\
\hline $600 \mathrm{rpm}$ & 0,1563 \\
\hline $900 \mathrm{rpm}$ & 0,1784 \\
\hline
\end{tabular}

Os valores de $\beta 0$ foram próximos, o que indica processos similares. No entanto, destaca-se o processo de aglomeração utilizando-se frequência de pulsação a $300 \mathrm{rpm}$, em que se obteve o maior valor de $\beta 0$. Neste caso, o valor de $\beta 0$ indica que processamento a $300 \mathrm{rpm}$ produz grânulos maiores, ou que o produto apresenta maiores frações de grânulos grossos - superior a $50 \mu \mathrm{m}$.

As figuras 4, 5 e 6 mostram a distribuição de tamanho obtida pelo modelo de balanço populacional para as diferentes frequências de pulsação. Observa-se que o modelo teve ajuste razoável, porém se pode verificar que o modelo de balanço populacional gera distribuições de tamanho próximas a uma curva Gaussiana e, por outro lado, a distribuição de tamanho experimental é melhor descrita por modelos Rosim-Ramler. 
Embora neste trabalho utilizaram-se diversos kernels de coalescência para a tentativa de melhor ajuste, em nenhum dos modelos a modelagem por balanço populacional gerou distribuições que se aproximam ao modelo de balanço populacional.

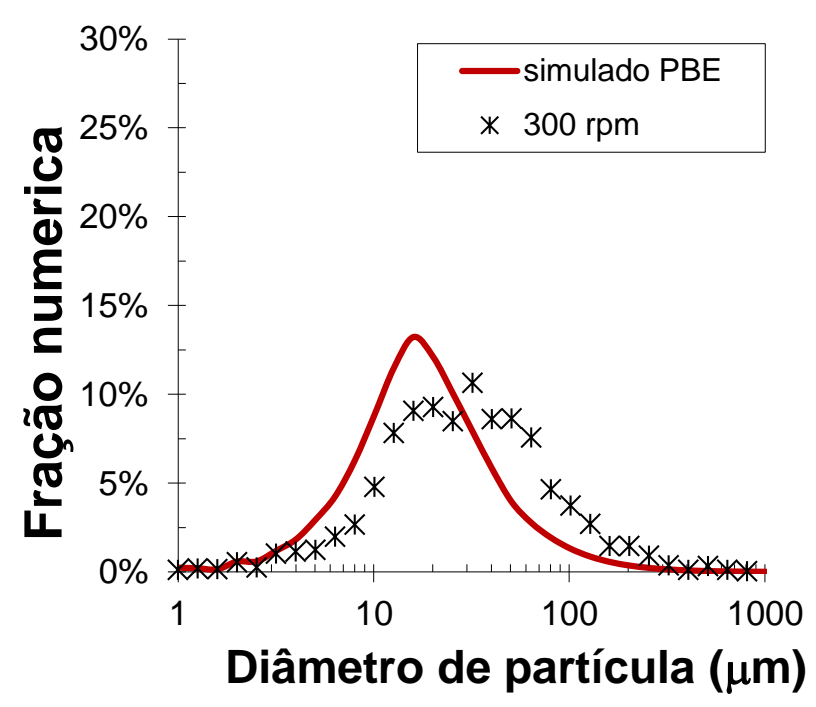

Figura 4 - Comparação entre a distribuição de tamanho de partículas para os grânulos produzidos a $300 \mathrm{rpm}$ e os resultados simulados pelo modelo de balanço populacional.

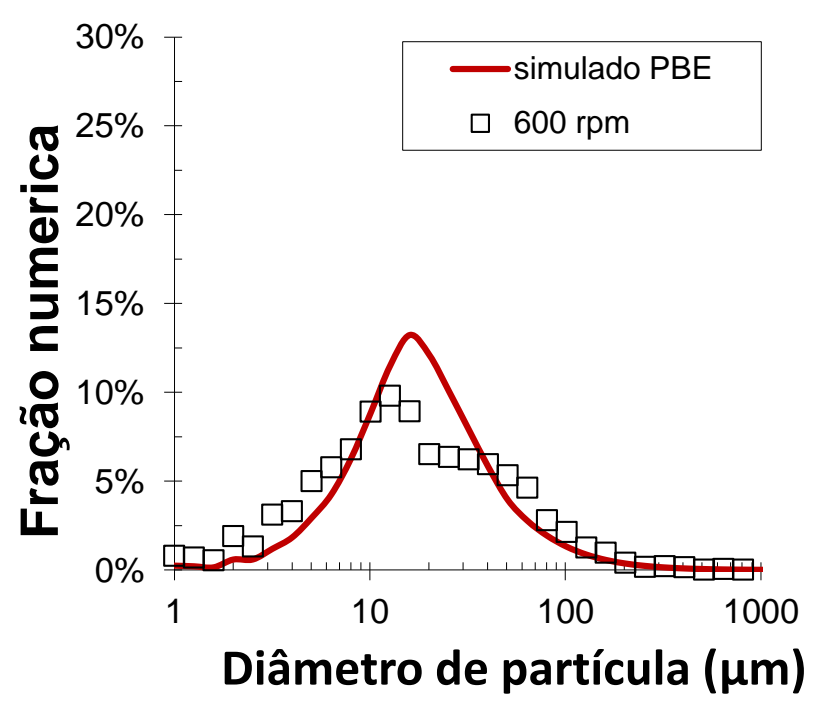

Figura 5 - Comparação entre a distribuição de tamanho de partículas para os grânulos produzidos a $600 \mathrm{rpm}$ e os resultados simulados pelo modelo de balanço populacional. 


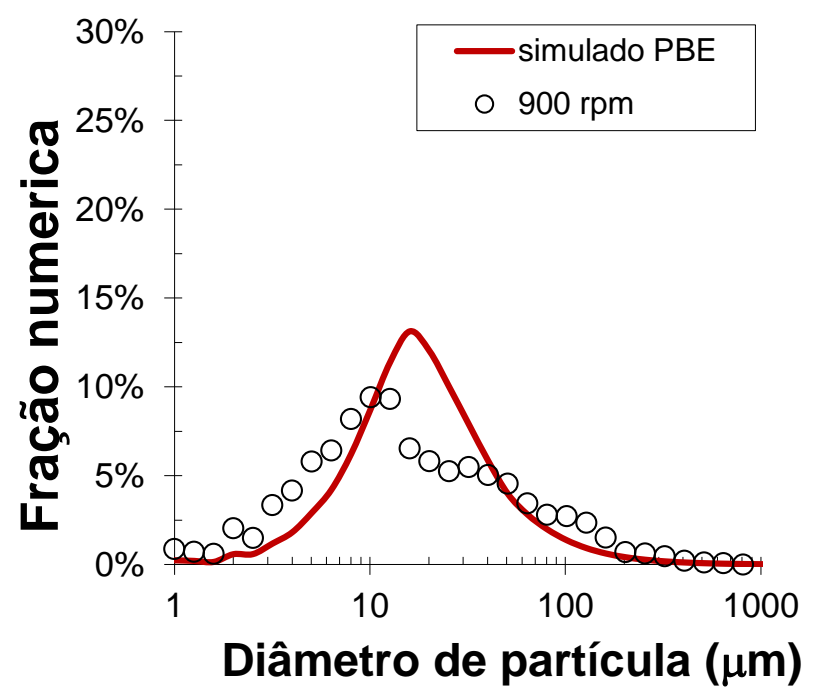

Figura 6 - Comparação entre a distribuição de tamanho de partículas para os grânulos produzidos a $900 \mathrm{rpm}$ e os resultados simulados pelo modelo de balanço populacional.

\section{CONCLUSÃO}

A modelagem por balanço populacional possibilita a extração de valores de $\beta 0$ e indica quais processos possuem maiores taxas de aglomeração, neste trabalho a maior taxa de aglomeração foi obtida a $300 \mathrm{rpm}$, apresentando um valor de $\beta 0$ igual a $0,1958 \mathrm{~min}^{-1}$. Porém, observa-se que os valores simulados pelo modelo de balanço populacional ainda possuem grandes desvios em relação à distribuição de tamanho obtida experimentalmente.

\section{AGRADECIMENTOS}

O presente trabalho foi realizado com o apoio financeiro da FAPESP (2010/18704-6).

\section{NOMENCLATURA}

$\begin{array}{lll}\beta_{0} & \text { Constante do modelo (kernel) } & {[-]} \\ d p_{e q} & \text { Diâmetro equivalente } & {[\mu \mathrm{m}]} \\ D 4.3 & \text { Diâmetro médio de Brouckere } & {[\mu \mathrm{m}]} \\ S & \text { Área de projeção 2D } & {\left[\mu \mathrm{m}^{2}\right]} \\ f_{i} & \text { Fração numérica } & {[-]}\end{array}$




\section{REFERÊNCIAS}

DACANAL, G.C.; MENEGALLI, F.C., Selection of operational parameters for the production of instant soy protein isolate by pulsed fluid bed agglomeration, Powder Technology, 203 (3), pp. 565-573, 2010.

HOUNSLOW, M.J.; PEARSON, J. M. K.; INSTONE, T. Tracer Studies of High-Shear Granulation: II. Population Balance Modeling. AIChE, v.47, v.9, p.1984-1999, 2001.

HOUNSLOW, M. J.; RYALL, R. L.; MARSHALL, V. R. A Discretized Population Balance for Nucleation, Growth, and Aggregation, AIChE Journal, v.34, n.11, p.1821-1832, 1988.

LIU, L.X.; LITSTER, J. D.; Population balance modelling of granulation with a physically based coalescence kernel. Chemical Engineering Science, v.57, p.2183-2191, 2002.

PEGLOW, M.; KUMAR, J.; HEINRICH, S.; WARNECKE, G.; TSOTSAS, E.; MORL, L.; WOLF, B. A generic population balance model for simultaneous agglomeration and drying in fluidized beds, Chemical Engineering Science, v.62, p.513-532, 2007.

RAMKRISHNA, D. Population Balances: Theory and Applications to Particulate Systems in Engineering, San Diego: Academic Press, 2000. 365p.

TAN, H. S.; SALMAN, A. D.; HOUNSLOW, M. J. Kinetics of fluidized bed melt granulation IV: Selecting the breakage model. Powder Technology, v.143, p.65 -83, 2004.

TAN, H. S.; SALMAN, A. D.; HOUNSLOW, M. J. Kinetics of fluidized bed melt granulation II: Modelling the net rate of growth. Chemical Engineering Science, v.61, p.3930-3941, 2006. 